\title{
Polymer dynamics in nanoconfinement: Interfaces and interphases
}

\author{
Margarita Krutyeva ${ }^{a}$, Andreas Wischnewski and Dieter Richter \\ Jülich Centre for Neutron Science, Forschungszentrum Jülich (JCNS-1) and Institute for Complex Systems (ICS), \\ Forschungszentrum Jülich GmbH, 52425 Jülich, Germany
}

\begin{abstract}
The dynamics of polymers in nanoconfinement was studied by using neutron spectroscopy. A number of pronounced effects on different time and length scales for the polymers confined in nanopores of anodic aluminium oxide were observed. Local segmental dynamics was found to be dependent on the type of the interaction between the solid pore wall and polymer: attractive interactions lead to the formation of a surface layer with the dynamics slowed down as compared to the dynamics of pure polymer; neutral/repulsive interaction do not change the local dynamics. Attractive interactions cause anchoring of polymer segments on the surface creating an interphase between the polymer in close vicinity to the solid surface and pure polymer. In addition, at strong confinement conditions the dilution of the entanglement network is observed.
\end{abstract}

\section{Introduction}

The last several years a dramatic increase in the synthesis of new nanoporous materials is observed [1]. They find a lot of unique applications in nanotechnology, chemistry and biochemistry. From an application perspective, the dominance of surfaces in nanoporous systems makes them attractive as matrices for gas storage, gas separation, catalysis and energy harvesting. Furthermore, nanoporous materials can be used as a step in the fabrication of multifunctional nanostructures or as membranes in separation processes [2,3]. In particular, nanostructured materials based on organic polymers led to the development of new miniature devices that exhibit unique capabilities not available in larger scale devices [4]. The majority of the applications are related to the structural changes and transport properties of the polymer embedded in the nanopores.

With respect to the structure of polymer chains in polymer films, it has been suggested that only the chain extension perpendicular to the film surface is depressed below its bulk value, whereas the parallel component remains unaltered [5]. However, the screening of intrachain excluded volume interactions progressively diminish with decreasing film thickness resulting in a chain swelling in direction perpendicular to the interfaces [6]. In contrast, in cylindrical nanopores the absence of chain anisotropy in this configuration was recently observed [7].

Microscopic studies of the dynamics on the chain level are mainly available through simulations, while only a few experimental studies on the chain dynamics under confinement can be found. In particular, physical adsorption of polymer chains on the surface of nanoparticles was reported that led to the formation of an glassy layer on the particle [8]. The simultaneous slowing down of Rouse modes, i.e. increasing of the friction coefficient, was found for unentangled polymer melts [9]. The combined effects of increased effective friction and entanglement

\footnotetext{
${ }^{a}$ Corresponding author: m.krutyeva@fz-juelich.de
}

mesh sizes were even found to lead to a non-monotonic behaviour of the chain relaxation with confinement size [10]. The strong confinement effect (so-called corset effect) was concluded from NMR relaxometry studies performed on poly(ethylene oxide) confined in solid methacrylate matrix. A reduction of the effective tube diameter of the Doi-Edwards reptation model in confined polymer by about one order of magnitude as compared to the bulk has been reported [11]. These results have been suggested to be the consequence of a number of combined effects: polymer-surface interaction, the mutual uncrossability of polymer chains (entanglements) and confinement geometry.

In this paper we present a review of recent microscopic investigations of the dynamics of different polymer melts embedded in nanopores of anodic aluminium oxide (AAO) or Alumina nanopores by means of neutron scattering.

\section{Model systems and methods}

Alumina nanopores have attracted increasing interest due to their anisotropic pore arrangement [12]. This is attractive from both, a fundamental and an applied point of view: They are ideal systems for the study of size-dependent properties. Moreover, Alumina nanopores are extremely versatile for creating different polymer multifunctional nanostructures, which makes them highly attractive for a variety of applications in nanotechnology. Well-ordered arrangement of the Alumina nanopores allows to access the anisotropic properties of the confined polymer. A typical scanning electron micrographs (SEM) of the nanopore structure are presented in Fig. 1. The inner surface of Alumina nanopores is covered by hydroxyl groups resulting in hydrophilic surface [13].

Infiltration of the polymer melts in the Alumina nanopores was performed by wetting-based methods $[4,14]$. Some characteristics of the samples are presented in Table 1. The melting temperature of the bulk

This is an Open Access article distributed under the terms of the Creative Commons Attribution License 4.0, which permits unrestricted use, distribution, and reproduction in any medium, provided the original work is properly cited. 
Table 1. Weight averaged molecular weight $M_{\mathrm{w}}$, characteristic end-to-end distance $R_{\text {ee }}$ pore diameter $D_{\mathbf{p}}$ and ratio $R_{\text {ee }} / D_{\mathbf{p}}$.

\begin{tabular}{|l|l|l|l|l|}
\hline Polymer & $M_{w}, \mathrm{~kg} / \mathrm{mol}$ & $R_{e e}, \mathrm{~nm}$ & $D_{p}, \mathrm{~nm}$ & $R_{e e} / D_{p}$ \\
\hline PEO-1 & 43 & 19 & 40 & 0.5 \\
\hline PEO-2 & 23 & 14 & 35 & 0.4 \\
\hline PEO-3 & 445 & 60 & 25 & 2.4 \\
\hline PEP & 29 & 16 & 20 & 0.8 \\
\hline PDMS & 17 & 8.5 & 26 & 0.3 \\
\hline
\end{tabular}

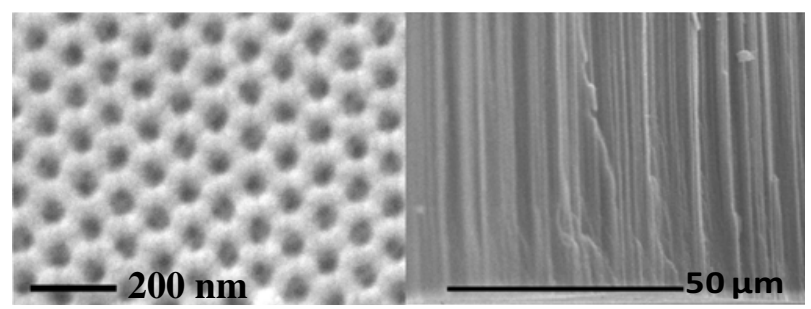

Figure 1. SEM micrographs of the surface (a) and cross section (b) of the Alumina nanoporous templates with pore diameter $D_{\mathbf{p}}=40 \mathrm{~nm}$.

poly(ethylene oxide) (PEO) is $338 \mathrm{~K}$, and its glass transition temperature ranges from $220 \mathrm{~K}$ to $228 \mathrm{~K}$, slightly dependent on molecular weight $M_{w}$. Polyethylene-altpropylene (PEP) does not crystallize and its $T_{g}$ is equal to $213 \mathrm{~K}$. Polydimethylsiloxane (PDMS) is a crystalline polymer with a melting point near $233 \mathrm{~K}$ and a glass transition temperature $T_{g}$ near $148 \mathrm{~K}$. The temperatures of the neutron scattering experiments presented in this paper were chosen to be higher than the crystallization temperature $T_{\mathrm{m}}$ and the glass transition temperature $T_{g}$ of the bulk polymers. The ratio of end-to-end distance $R_{e e}$ of the polymer chain to the pore diameter $D_{p}$ characterizes the so-called confinement condition. In particular, weak $\left(R_{\mathrm{ee}} D_{\mathbf{p}}<1\right)$ and strong $\left(R_{\mathrm{ee}} D_{\mathbf{p}}>1\right)$ confinement conditions will be discussed below.

Neutron scattering gives direct access to dynamical properties with space/time resolution. This allows separating different dynamical regimes on different length scales [15]. Incoherent quasielastic neutron scattering experiments with PEO were performed to study the local segmental dynamics in the sample PEO-1. Later the dynamics on larger time scales was measured by neutron spin echo (NSE) on samples PEO-2 and PEO-3.

\section{Influence of polymer-surface interaction on the local polymer dynamics}

\subsection{Case of attractive surface}

Polyethylene(oxide) is considered as a good candidate to demonstrate the influence of the attractive surface on the polymer dynamics. To study the local dynamics the timeof-flight (TOF) and backscattering (BS) techniques were used [16].

In the momentum transfer range usually explored by the TOF and BS $0.2 \AA^{-1}<\mathrm{Q}=|\vec{Q}|<1.9 \AA^{-1}$ the decay of the incoherent intermediate scattering function $S_{\text {inc }}(Q, t)$

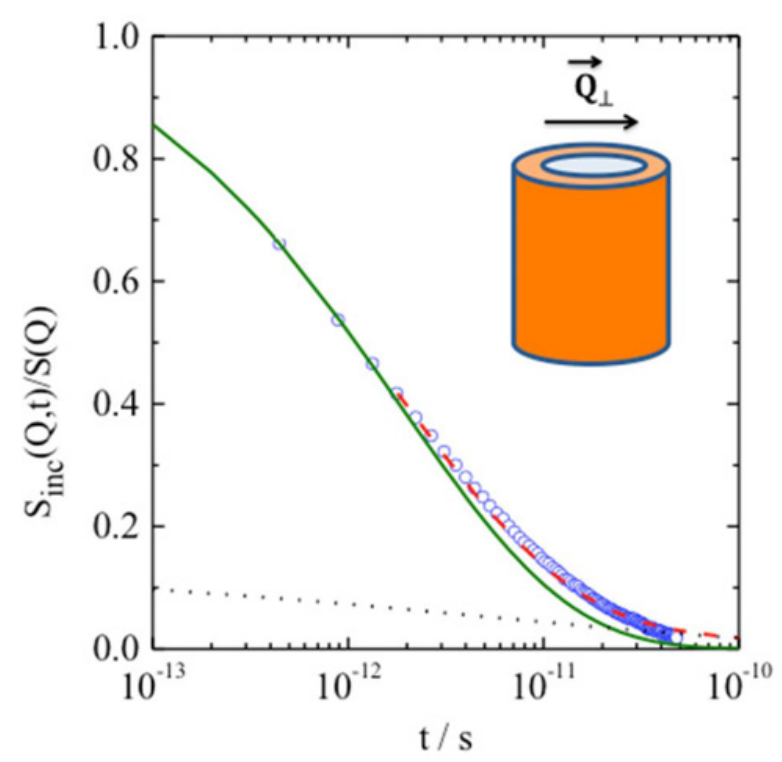

Figure 2. Intermediate scattering function of PEO-1 (circles) in the Alumina nanopores, measured perpendicular to the pore axis and the respective function for bulk PEO (solid green line) [17] at $375 \mathrm{~K}$ and $\left|\vec{Q}_{\perp}\right|=1.4 \AA^{-1}$. The dashed line represents a fit by a sum of $87 \%$ of the fast bulk component and $13 \%$ slow component. The slower component is shown by the dotted line. In the insert a sketch of the cylindrical Alumina nanopore is presented.

is analysed by a sum of two empirical stretched exponential or Kohlrausch-Williams-Watts (KWW) functions:

$$
\begin{aligned}
S_{\text {inc }}(Q, 1)= & A_{\text {bulk }}(Q) \exp \left(-\left(\frac{t}{\tau_{b u l k}^{K W W}}\right)^{\beta_{b u l k}}\right) \\
& +\left(1-A_{b u l k}(Q)\right) \exp \left(-\left(\frac{t}{\tau_{\perp}^{K W W}}\right)^{\beta_{\perp}}\right)
\end{aligned}
$$

where $\tau_{\perp}^{K W W}$ and $\tau_{\text {bulk }}^{K W W}$ are the relaxation times associated with the hydrogen displacements perpendicular to the pore axis and in a bulk, respectively. $\beta_{\perp}$ and $\beta_{\text {bulk }}=0.5$ are stretching parameters for the confined perpendicular polymer fraction and bulk PEO. The relaxation time obtained for the parallel component is equivalent to the bulk $[16,17]$. In particular, in the Fig. 7 of the Ref. [16] $Q$-dependence of the relaxation time $\tau_{\perp}^{K W W}$ for different temperatures for bulk and confined PEO is presented.

Dynamics of the PEO in the direction perpendicular to the pore walls is determined by two fractions. One is attributed to the bulk and the second fraction represents the surface layer, where the dynamics of the latter is slowed down as compared to the bulk value. It is remarkable that the surface layer is not glassy or immobile in a broad temperature range from $300 \mathrm{~K}$ to $492 \mathrm{~K}$.

\subsection{Case of neutral/repulsive surface}

Neutral or repulsive interaction between the polymer segments and the Alumina surface is expected for polyethylene-alt-propylene. In this case the polymer chain 


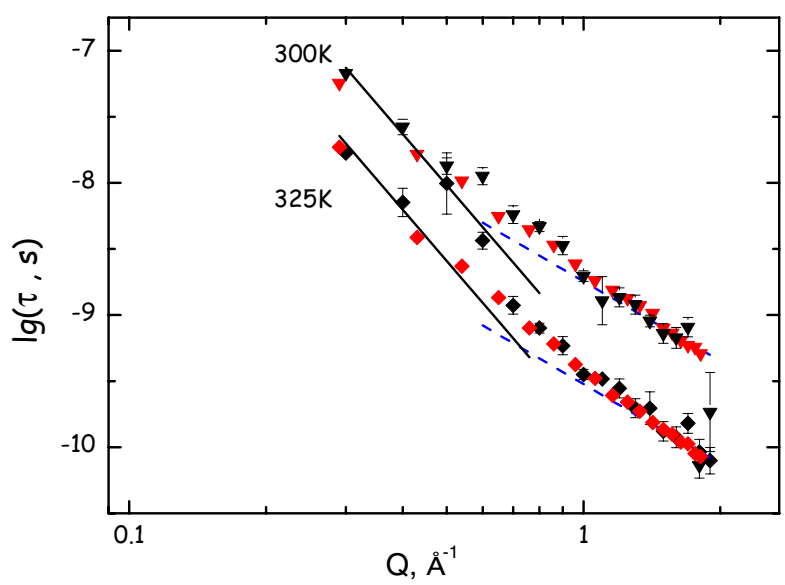

Figure 3. Characteristic relaxation times of PEP in Alumina nanopores oriented perpendicular to the pore axis $\left(\tau=\tau_{\perp}\right)$ (black symbols), and in the bulk (red symbols, [18]) as a function of the momentum transfer $\mathrm{Q}$ for different temperatures. The solid line indicates a slope of $Q^{-4}$ and the dashed line a slope of $Q^{-2}$.

is a pure hydrocarbons and therefore does not form hydrogen bonds with the hydroxyl groups on the Alumina surface. Thus, it is expected that the polymer-surface interaction will not essentially influence the dynamical properties of the polymer chain. Indeed, the BS and TOF spectra are equivalent in the pore and in the bulk. Further analysis in terms of KWW functions demonstrates no change in the dynamics as compared to unconfined PEP [18]. It is remarkable that even deviation from Gaussian behaviour $\tau \propto Q^{-2 / \beta}$ with $\beta=$ 0.5 is reproduced in confinement and in large $Q$ regime represented by power law $Q^{-2}$.

In Fig. 3 the relaxation times for PEP confined in Alumina nanopores and pure PEP are presented. From the figure it is evident that the absence of attractive forces does not influence the local segmental dynamics of polymer segment.

\section{Confinement effects on the polymer matrix}

As it was shown, the local segmental dynamics is influenced by polymer-surface interactions. At longer times it may lead to the specific effects on the large-scale dynamics of polymer chain that can be well studied by the NSE technique [15].

Polymer chain dynamics is commonly described in terms of the Rouse and the reptation model. The relaxation of the Rouse modes, determined by a balance of viscous and entropic forces, only depends on the monomeric friction. In addition, long polymers heavily interpenetrate each other and mutually restrict their motions at long times in forming topological constraints ("entanglements") [19]. The important question that is addressed both by computer simulations $[9,10]$ as well as by a variety of experiments on a macroscopic level [20-22] is how the dynamics in the Rouse and reptation regime change under confinement.

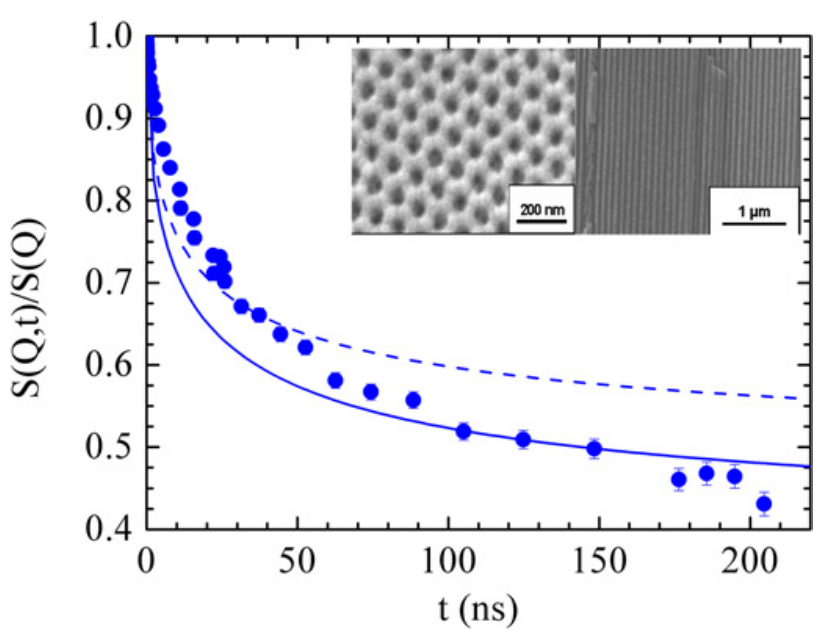

Figure 4. Normalised dynamical structure factor of single polymer chain in strong confinement measured at $Q=0.1 \AA^{-1}$ and $400 \mathrm{~K}$. The dashed line illustrates designates the reptation model predictions with an effective tube diameter of the PEO polymer chain in the bulk, $d_{\text {bulk }}=53 \AA$. The solid line presents a fit with the reptation theory yielding a larger value for the effective tube diameter, $d_{\text {conf }}=60.3 \AA$ indicating a dilution of the entanglement network. In the inset, the scanning electron micrograph of the nanoporous Alumina is presented.

\subsection{Entanglement network under confinement}

Here the results on the single chain dynamic structure factor of PEO confined in Alumina nanopores for the chain dimensions $R_{\mathrm{ee}}$ either much larger or smaller than the pore diameter $D_{\mathrm{p}}$ (samples PEO-2 and PEO-3) at $400 \mathrm{~K}$ are presented. The samples were partially deuterated, and then contrast-matched to the porous Alumina whose scattering strength had been determined in a previous SANS experiment [23]. In this way, the scattering due to the motions of the polymer chains could be highlighted.

At long times well beyond the entanglement limit ( $t>\tau_{\mathrm{e}}$ where $\tau_{\mathrm{e}}$ is characteristic entanglement time), for $R_{\text {ee }} / D_{\mathbf{p}}>1$ we found a $15 \%$ expanded entanglement network (e.g. effective tube diameter $d$ in the reptation model [15]), while for $R_{\mathbf{e e}} / D_{\mathbf{p}}<1$ the bulk values are reproduced in agreement with computer simulations and other experiments [9, 10,20-22].

As compared to the bulk PEO, a moderate slowing down of the dynamics in the intermediate time regime was noticed (Fig. 4). This effect, other than simulations suggested [10], may not be related to a general increase of the Rouse friction, since the local dynamics at short times is found to be largely unchanged.

One possible explanation for the observed behaviour could be some adsorption effect on the surface of the pore walls. The alumina surface "attracts" hydrophilic PEO chains, leading to a complete wetting of the AAO surface by the polymer melt. In a first approximation the adsorption effect was modelled by a Rouse chain with pinned segments, such that on average the surface layer is covered [23]. Although the results of this approximation are in qualitative agreement with the NSE data for the PEO, one cannot exclude the influence of the 


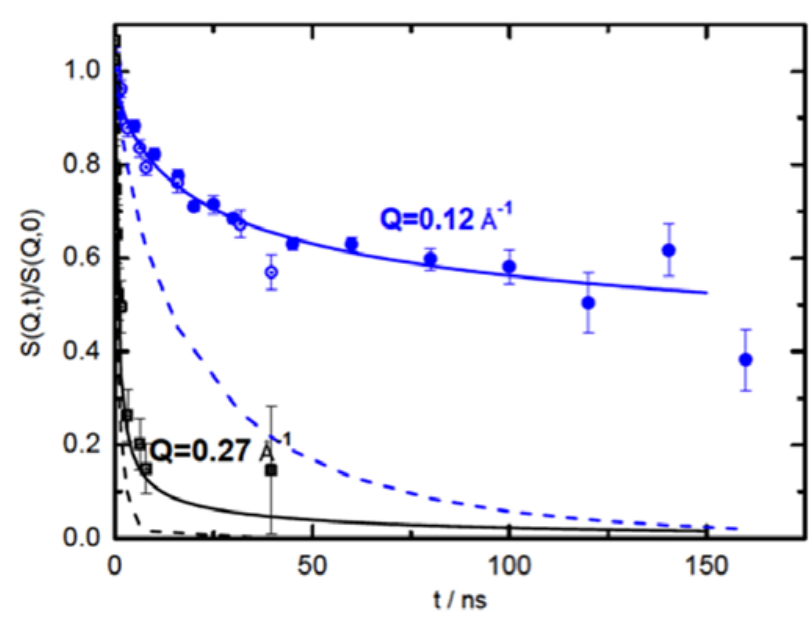

Figure 5. NSE data (symbols) obtained for the confined PDMS at two different $Q$-values (shown in the figure) and at $373 \mathrm{~K}$. Solid lines present a fit with the two-phase suppressed Rouse model (Eq. (2)). Dash lines show the calculated dynamical structure factor of the bulk PDMS.

entanglements on time scales larger than $10 \mathrm{~ns}$. Similar to the adsorption, entanglements slow down dynamics on the intermediate timescale and discrimination of the two phenomena is a challenge.

\subsection{Interphase formation}

To investigate the Rouse dynamics under confinement on an intermediate timescale polydimethylsiloxane was used. PDMS has relatively large entanglement molecular weight $M_{e}=12 \mathrm{~kg} / \mathrm{mol}$ that allows to extend the dynamical range of NSE where the entanglement effects are negligible. PDMS with a molecular weight a factor 1.5 larger than the entanglement molecular weight was infiltrated into Alumina templates with the pore diameter $26 \mathrm{~nm}$ (Table 1). Calculations using density functional theory showed an adsorption interaction between the Alumina surface and PDMS.

The NSE data obtained at $373 \mathrm{~K}$ show a significantly slowed down chain dynamics compared to the bulk (Fig. 5). Proper inspection of the plateaus measured at different values of the momentum transfer $Q$ (some data are not presented here) at long times made it possible to exclude the existence of an immobilized (glassy) layer, since the plateaus are $Q-$ dependent. Note, that this result is in agreement with our previous data on PEO confined in Alumina nanopores [16] and with a recent neutron scattering study [24]. Furthermore, the observed $Q$-dependence of the plateaus cannot be described in terms of a simple confined polymer, i.e. one-phase system, because fitting such a model to the data yields a $Q$-dependent confinement length.

Therefore, a new model was derived containing (i) a fraction of free Rouse-like chains, since many chains far away from the surface are not affected and (ii) a second fraction of chains, which is close to the surface and assumed to be effectively confined. The respective

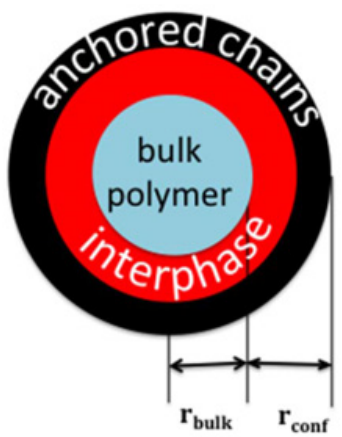

(a) (b)

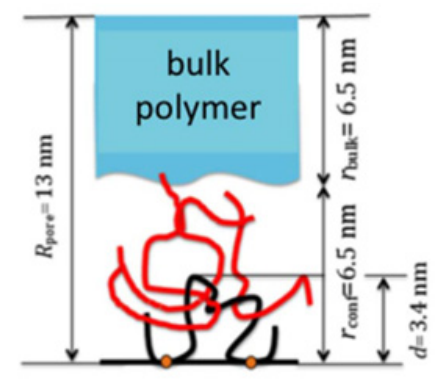

Figure 6. Schematic representation of the surface-induced entanglements in the confined polymer melt in cylindrical nanopore (a) and perpendicular to the pore surface (b). The chains adsorbed on the surface (anchored chains) are shown by black colour, and the entangled chains in the confined phase (interphase) are represented by red colour.

dynamic structure factor reads:

$$
S(Q, t)=A_{\text {bulk }} S_{\text {bulk }}(Q, t)+A_{\text {conf }} S_{\text {conf }}(Q, t)
$$

where $A_{\text {bulk }}$ and $A_{\text {conf }}=1-A_{\text {bulk }}$ are the fractions of the bulk and confined phases, respectively; $S_{\text {bulk }}(Q, t)$ is the dynamic structure factor of the bulk chain defined by the Rouse model [25] and $S_{\text {conf }}(Q, t)$ is the dynamic structure factor of the confined chain. This model contains three variable parameters: fraction $A_{\text {bulk }}$ (or $A_{\text {conf }}=1-$ $\left.A_{\text {bulk }}\right)$, the number of suppressed Rouse modes $p_{\text {supp }}$ reflecting the effective confinement in $S_{\text {conf }}(Q, t)$ and an additional parameter $\Delta p$, the width of a cut off function describing the transition from active to suppressed modes. $\Delta p$ accounts for a variety of anchoring and confinement conditions. By fitting all $Q$ simultaneously $A_{\text {bulk }}$ was found to be $25 \%$ of the total amount of polymer in the pores, $p_{\text {supp }}=5$ and $\Delta p=1$ (Fig. 5). From the longest active mode in the confined phase one can calculate the effective confinement length $d=3.4 \mathrm{~nm}$.

The fractions $A_{\text {bulk }}$ and $A_{\text {conf }}$ obtained can be transferred to a corresponding layer thickness which was found to be $r_{\text {conf }}=r_{\text {bulk }}=6.5 \mathrm{~nm}$ (Fig. 6). The fact that the size of the PDMS polymer subchain between the anchored points is $3.4 \mathrm{~nm}$ clearly indicates that the layer with a thickness of $6.5 \mathrm{~nm}$ is not only made up of chains adsorbed at the surface. The polymer chains anchored to the surface may form loops; neighbouring chains can then interpenetrate these loops (Fig. 6). These penetrating chains can be considered as an interphase between the polymers adsorbed on the surface and the bulk polymer phase.

Formation of loops and also trains and tails by the polymer chains on the attractive surface was observed by computer simulations [26] and theory [27,28]. In particular, it was found that the loop distribution follows a power law relationship.

In general, the confined phase composed of the anchored chains and the chains in the interphase is internally highly mobile and not glassy as has so far been claimed frequently in the literature. These results are inferred from the space-time dependent chain dynamics 
that is observed in terms of the single chain dynamic structure factor and represent the first direct and quantitative observation of the interphase [29].

\section{Conclusions}

In this study we reviewed our recent results on the effects of nanoconfinement on the dynamics of polymer melts obtained by means of neutron scattering. We focussed in particular on the influence of the polymersurface interaction on the dynamics of polymer confined in cylindrical nanopores of anodic aluminium oxide. As expected, the local dynamics does not change if interactions between the polymer segment and pore wall is neutral or repulsive. On the other side, attractive interactions lead to the adsorption of polymer segments on the Alumina surface through the bond formation with hydroxyl groups. This results in some significant dynamical effects. First, a polymer layer with a thickness $1 \mathrm{~nm}$ is formed in close vicinity to the surface. The dynamics of this surface layer is slower than the dynamics of pure polymer. On the time- and length scale of a polymer chain the dynamics was found to be nondistinguishable from the dynamics of pure polymer in the case of a weak confinement. At strong confinement conditions a dilution of the entanglement network was discovered. Finally, we demonstrate that the attractive polymer-surface interaction leads to the formation of an interphase in a confined polymer melt.

We thank the instrument responsible at the neutron facilities: P. Falus at ILL (Grenoble, France), O. Holderer, G.J. Schneider, T. Unruh at MLZ (Garching, Germany) and N. Jalarvo, M. Ohl at the SNS ORNL (Oak Ridge, USA) who contributed to all performed experiments, J. Allgaier and L. Willner for polymer synthesis, J. Martín, J. Maiz and C. Mijangos for Alumina template synthesis and samples preparation, M. Monkenbusch, A. Arbe, J. Colmenero for fruitful discussions. The support of the DFG Priority Program SPP1369 Polymer-Solid Contacts: Interfaces and Interphases is gratefully acknowledged.

\section{References}

[1] Nanoporous materials: Advanced techniques for characterization, modelling and processing, edited by N. Kanellopoulos (CRC Press Taylor \& Francis Group, 2011)

[2] A. Vaseashtaa, D. Dimova-Malinovska, Sci. Technol. Adv. Mat. 6, 312 (2005)

[3] P. Stroeve, N. Ileri, Trends Biotechnol. 29 (6) 259 (2011)

[4] J. Martín, J. Maiz, J. Sacristan, C. Mijangos, Polymer 53, 1149 (2012)

[5] A. J. Silberberg, J. Colloid Interf. Sci. 90, 86 (1982)

[6] H. Meyer, T. Kreer, A. Cavallo, J. P. Wittmer, J. Baschnagel, Eur. Phys. J. Spec. Top. 141, 167 (2007)
[7] L. Noirez, C. Stillings, J.-F. Bardeau, M. Steinhart, S. Schlitt, J. H. Wendorff, and G. Pépy Macromolecules 46, 4932 (2013)

[8] S.Y. Kim, H.W. Meyer, K. Saalwächter, and C. F. Zukoski, Macromolecules 45, 4225 (2012)

[9] P. J. Dionne, R. Ozisik, and C. R. Picu Macromolecules 38, 9351 (2005)

[10] Y. Li, D. Wei, C. C. Han, Q. Liao, J. Chem. Phys. 126, 204907 (2007)

[11] N. Fatkullin, R. Kimmich, E. Fischer, C. Mattea, U. Beginn, and M. Kroutieva, New J. Phys. 6, 46 (2004)

[12] H. Masuda, K. Fukuda, Science 268 (5216), 1466 (1995)

[13] M. Digne, P. Sautet, P. Raybaud, P. Euzen, H. Toulhoat, J. Catal. 211, 1 (2002)

[14] M. Zhang, P. Dobriyal, J-T. Chen, T.P. Russel, J. Olmo, A. Merry, Nano Lett. 6 (5), 1075 (2006)

[15] D. Richter, M. Monkenbusch, A. Arbe, J. Colmenero, Adv. Polym. Sci. 174, 1 (2005)

[16] M. Krutyeva, J. Martin, A. Arbe, J. Colmenero, C. Mijangos, G. J. Schneider, T. Unruh, Y. Su, D. Richter, J. Chem. Phys. 131, 174901 (2009)

[17] M. Brodeck, F. Alvarez, A. Arbe, F. Juranyi, T. Unruh, O. Holderer, J. Colmenero, and D. Richter, J. Chem. Phys. 130, 094908 (2009)

[18] R. Pérez Aparicio, A. Arbe, J. Colmenero, B. Frick, L. Willner, D. Richter, L. J. Fetters, Macromolecules 39, 1060 (2006)

[19] M. Doi and S. Edwards, The Theory of Polymer Dynamics (Clarendon, Oxford, 1986)

[20] L. Si, M.V. Massa, K. Dalnoki-Veress, H. R. Brown, R. A. L. Jones, Phys. Rev. Lett. 94, 127801 (2005)

[21] O. Bäumchen, R. Fetzer, K. Jacobs, Phys. Rev. Lett. 103, 247801 (2009)

[22] K. Nusser, G. J. Schneider, D. Richter, Macromolecules 44, 5857 (2011)

[23] J. Martin, M. Krutyeva, M. Monkenbusch, A. Arbe, J. Allgaier, A. Radulescu, P. Falus, J. Maiz, C. Mijangos, J. Colmenero, D. Richter, Phys. Rev. Lett. 104, 197801 (2010)

[24] T. Glomann, G. J. Schneider, J. Allgaier, A. Radulescu, W. Lohstroh, B. Farago, D. Richter, Phys. Rev. Lett. 110, 178001 (2013)

[25] P. J. Rouse, J. Chem. Phys. 21, 1272 (1953)

[26] A. De Virgiliis, A. Milchev, V.G. Rostiashvili, T.A. Vilgis, Eur. Phzs. J. E 35: 97 (2012)

[27] J.M.H.M. Scheutjens, G.J. Fleer, J. Phys. Chem. 84, $178(1980)$

[28] C.A.J. Hoeve, E.A. DiMarzio, P.Peyser, J.Chem. Phys. 42, 2558 (1965)

[29] M. Krutyeva, A. Wischnewski, M. Monkenbusch, L. Willner, J. Maiz, C. Mijangos, A. Arbe, J. Colmenero, A. Radulescu, O. Holderer, M. Ohl, D. Richter, Phys. Rev. Lett. 110, 108303 (2013) 